\title{
CHEMISTRY OF SOME NATURAL PIGMENTS
}

$\mathbf{A}^{\mathrm{T}}$

T. the meeting of the British Association this year in Oxford, a symposium was held on September 6 on "Complex Natural Colouring Matters". After a short introduction by Sir Robert Robinson, Sir Alexander Todd gave a lucid account of the recent work which has been carried out in the Cambridge laboratory on the structure of aphins, the pigments obtained from the hæmolymph of various species of Aphidiae. For each species so far examined, the following four types of compounds have to be considered : the water-soluble protoaphins, which are probably glycosides and represent the 'native' precursor of the pigments; the fat-soluble xanthoaphins which are formed after the death of the insect by enzymic action; the orange chrysoaphins representing an intermediate state in the transformation chain; and the deep-red erythroaphins which represent the relatively stable end-products of each series. The two series which have so far been examined in detail are the aphins- $f b$ obtained from Aphis fabae, the blackfly which attacks broad beans, and the aphins-sl, derived from Tuberolachnus salignus, the willow aphid. The contribution of Sir Alexander Todd was concerned entirely with the structures of erythroaphin-fb and erythroaphin-sl. Papers already published in the Journal of the Chemical Society have shown that both these two substances have the formulæ $\mathrm{C}_{30} \mathrm{H}_{22} \mathrm{O}_{8}$, that they contain two carbonyl groups (quinone) and two hydroxyl groups, while the remaining four oxygen atoms are inert and are probably present in an ether linkage. The results of zinc dust distillation and of examination by infra-red and ultra-violet spectroscopy have shown that there is present in both compounds a $4: 9$-dihydroxyperylene-3:10-quinone structure. The structure now proposed for the two erythroaphins is based on the following arguments.

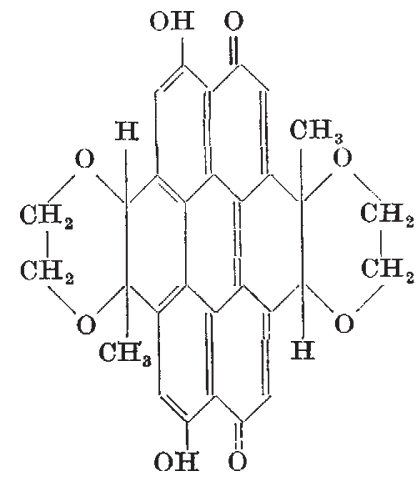

Substitution reactions show elearly that positions 2, 5, 8 and 11 in the perylene nucleus are free. Zinc dust fusion yields, among other products, a coronene derivative, suggesting that the saturated part of the molecule is attached to the perylene ring at positions 1 and/or 12 and 6 and/or 7. The formation of acetaldehyde on heating with hot 60 per cent sulphuric acid, and also symmetry considerations, make it appear probable that in these two erythroaphins two dioxan rings are fused with the central perylene nucleus.

Both erythroaphins are optically active, but their specifie relations differ markedly. From the fact that erythroaphin-fb yields $1 \cdot 7$ moles of acetaldehyde and erythroaphin-sl only 0.8 mole, it is deduced that the two alicyclic ring junctions in the former are both cis, whereas in the $s l$-isomer one junction is cis and the other is trans. This deduction is in accordance with modern concepts of the stereochemistry of elimination reactions and also provides a satisfactory explanation of some of the complex interconversion and isomerization reactions of the erythroaphins and their derivatives. Although the structures proposed may not be established with complete certainty, all the known facts, physical and chemical, fit beautifully this ingenious interpretation.

The second contribution, by Prof. R. P. Linstead, was mainly concerned with recent work carried out. in the Chemistry Department of the Imperial College of Science and Technology, London, on the chemistry of chlorophyll. This pigment, which plays an important part in photosynthesis in plants, is chemically related to the porphyrins; but, whereas the porphyrins are red and show five discrete absorption bands in the visible and near ultra-violet regions of the spectrum, chlorin, the basic macrocyclic structure present in chlorophyll, is green and is distinguished by a strong and sharp band in the red region. It has been shown, mainly by the work of the late Hans Fischer, that chlorins possess two more hydrogen atoms in the ring system than the corresponding porphyrins, and these additional hydrogen atoms are mainly responsible for the differences in colour and presumably of biological function of these two classes of complex organic molecules. The introduction of these two hydrogen atoms also creates a new element of asymmetry in the molecule and accounts for the optical activity of chlorophyll and its derivatives. Porphyrins are optically inactive, unless they acquire asymmetrical carbon atoms in their side-chains.

The placing of these two hydrogen atoms in the ring system proved to be a particularly difficult problem which was only solved in principle by Fischer in 1940 by oxidative degradation of various chlorophyll derivatives. Oxidation of porphyrins with chromic trioxide has been used for a long time in structural studies; thus with mesoporphyrin, which is related to the protoporphyrin of hæmoglobin, it produces two molecules of ethylmethylmaleimide, derived from the two non-acidic rings I and II, and two molecules of a maleimide propionic acid (hæmatinic acid), derived from the acidic rings III and IV. In 1940, Fischer and Wenderoth succeeded in isolating from a chlorophyll derivative in the form of a silver salt a dihydrohæmatinic acid which was optically active. This observation, together with other available knowledge, indicated that the two hydrogen atoms occupy the $\beta$-positions in ring IV. However, Fischer was unable, by the methods then available, to obtain the dihydro acid in a crystalline state, nor was it possible to determine the steric configuration of the compound.

Prof. Linstead and his colleagues have tackled the problem of the 'extra hydrogens' in three ways: in the first place (with Dr. M. Whalley), stable extra. hydrogen atoms have been introduced into tetra. methyl tetrazaporphin, which is a compound of related structure in which the four methene bridge groups are replaced by nitrogen. This azaporphin took up on catalytic hydrogenation four atoms of hydrogen. On oxidation, good yields of meso-1 : 2dimethyl-succinimide and dimethylmaleimide were obtained, indicating that the hydrogen atoms had 
indeed entered into the $\beta$-positions. This work also demonstrated that the tetrahydride structure involving the $\beta$-positions corresponds to a moderately stable reduction-level, at least in the azaporphin series.

Although porphin, the completely unsubstituted porphyrin, had been known for some time, the corresponding chlorin had so far not been synthesized. Prof. Linstead and Dr. U. Eisner have now obtained this substance in the following manner : pyrrole is converted by a Mannich reaction to 2-dimethylaminomethylpyrrole, and this on treatment with a Grignard reagent yields a complex mixture of pigments from which chlorin is isolated in low yield by counter-current distribution and chromatography. The light absorption of the chlorin is extremely close to that observed by Stern with the various natural chlorins supplied by Fischer. Chlorin also gives the correct analysis for a dihydride and can be converted to porphin.

Finally, Prof. Linstead described recent work with Dr. G. E. Fichen on dihydrohæmatinic acid. This compound possesses two dissimilar asymmetric carbon atoms and will therefore exist in two racemic forms. The anhydrides, the imides and various salts of both series have been prepared, and a relatively easy interconversion from one series to the other has been observed to take place. Natural dihydrohæmatinic acid has been prepared by oxidation of pyrrophæophorbide-a (a degradation product of chlorophyll a) and has been separated from methylethylmaleimide by chromatography on silica gel. The natural substance has $[\alpha]_{5893}=-51^{\circ}$. In respect of all other properties examined, it closely resembles one of the two racemic materials prepared by synthesis. The configuration of the two asymmetric carbon atoms has not yet been determined, and further developments of this work will be of great interest.

The third contribution, by Dr. A. Neuberger, was concerned with the biogenesis of porphyrins. After a short survey of their biological role, the structure of some of the naturally occurring porphyrins was briefly discussed and the point was emphasized that all the porphyrins associated with proteins are formally derivable from æetioporphyrin III, that porphyrins of type I are also found in Nature, while isomers of the series II and IV are apparently not synthesized by living matter.

From the wide distribution of cytochromes and catalase it can be deduced that almost all cells can synthesize porphyrins, but up to eight years ago there was no information as to the nature of the precursors. However, in 1946, Shemin and Rittenberg showed by means of labelling with heavy nitrogen that the nitrogen of glycine is used in a highly specific manner in the synthesis of protoporphyrin. Later work done in the United States by Shemin and in Britain by Muir and Neuberger produced convincing evidence that all four nitrogen atoms of porphyrin are specifically derived from glycine. When carbon-labelled glycine became available, it was shown by Altman that the carboxyl carbon atom of glycine is not incorporated into porphyrin, while parallel work done in the Columbia laboratories and at the National Institute for Medical Research, Mill Hill, demonstrated that the methylene carbon atom of glycine was used. In fact, for each nitrogen atom of glycine two methylene carbon atoms were found to be incorporated into each of the four basic units. By oxidative degradation it was found that one of these glycine methylene carbon atoms gives rise to the methene bridge, while the other is incorporated into the pyrrolic ring. Later, it was shown by Shemin that one of the two $\alpha$-carbon atoms originates from the second glycine methylene group. This, of course, accounts only for eight out of thirty-four carbon atoms of protoporphyrin. Experiments with carbon-labelled acetate carried out in Columbia and at Mill Hill suggest that $\alpha$-oxoglutarate and a substance closely related to it provide all these other carbon atoms; but it has been finally shown by Shemin that the substance concerned is mainly a succinic acid derivative substituted on one of the carboxyl groups, probably the coenzyme- $A$ ccmplex of this dicarboxylic acid. The position thus is that each of the four basic units of uroporphyrin can be thought to be formed by condensation of two molecules of glycine with two molecules of a succinic acid derivative and that one of the two nitrogens and the two carboxyl groups of glycine are lost before, during or after this condensation.

The identification of the intermediate between glycine plus succinic acid and the porphyrins has proved to be a difficult task and is by no means completely solved. Neuberger and Muir considered that the basic unit is a pyrrole substituted on one of the $\alpha$-positions with elements of a potential methene group. This may have been formed by an initial condensation of two glycine molecules followed by combination with succinic acid. However, work with substances such as $\beta$-hydroxy aspartic acid was discouraging, and this idea was discarded also for other reasons. If the basic pyrrole has already one of the two $\alpha$-positions substituted, it is easy to see how the symmetrical porphyrin of type I is formed, but the synthesis of the asymmetrical type III is more difficult to explain. A more reasonable assumption was that the basic pyrrole has two free $\alpha$-positions, and this pyrrole would then combine with a further molecule of glycine giving one or other of two different $\alpha$-substituted substances. It was therefore assumed that glycine would condense with succinic acid giving, in the first place, $\alpha$-amino $\beta$-oxoadipic acid which would then be converted by loss of carbon dioxide to $\delta$-aminolævulic acid. This acid might then react with a further molecule of succinic acid to give $\delta$-succinamidolævalie acid, which could be reduced to the hypothetical pyrrole. However, biological experiments showed that the succinamido compound is inactive.

In the meantime, Westall had crystallized porphobilinogen, a substance excreted by patients suffering from acute porphyria. Its structure has been established by Cookson and Rimington as a pyrrole having an acetic acid and a propionic acid side-chain in the two $\beta$-positions and an aminomethyl group in one of the two $\alpha$-positions. Soon afterwards Falk, Dresel and Rimington showed that porphobilinogen behaves as a porphyrin precursor, and it thus seems that the original idea of the Mill Hill workers may be partially correct and that an $\alpha$-monosubstituted pyrrole is the first cyclic intermediate in the synthesis. From the structure of porphobilinogen it can be inferred that $\delta$-aminolævulic acid itself might be the sought intermediate. This was shown almost simultaneously by Shemin and Russell and by Neuberger and Scott, and confirmed by Falk and Dresel. Recent work at Mill Hill, which was briefly reported by Dr. Neuberger, has greatly strengthened this concept that glycine and succinic acid combine to give $\delta$-aminolævulic acid, which gives rise to porphobilinogen and then to porphyrins. 